\title{
ELECTROWEAK MODEL AND CONSTRAINTS ON NEW PHYSICS
}

To reduce the size of this section's PostScript file, we have divided it into three PostScript files. We present the following index:

\section{PART 1}

\begin{tabular}{cll}
\hline Page \# & \multicolumn{2}{l}{ Section name } \\
\hline 1 & 10.1 & Introduction \\
2 & 10.2 & Renormalization and radiative corrections \\
6 & 10.3 & Cross-section and asymmetry formulas \\
\hline \hline
\end{tabular}

PART 2

\begin{tabular}{cll}
\hline Page $\#$ & \multicolumn{2}{l}{ Section name } \\
\hline 12 & 10.4 & $W$ and $Z$ decays \\
13 & 10.5 & Experimental results \\
\hline \hline
\end{tabular}

PART 3

\begin{tabular}{|c|c|}
\hline Page \# & Section name \\
\hline 23 & 10.6 Constraints on new physics \\
\hline 29 & References \\
\hline
\end{tabular}




\section{Electroweak model and constraints on new physics 1 10. ELECTROWEAK MODEL AND CONSTRAINTS ON NEW PHYSICS}

Revised October 1997 by J. Erler and P. Langacker (Univ. of Pennsylvania).
10.1 Introduction
10.2 Renormalization and radiative corrections
10.3 Cross-section and asymmetry formulas
$10.4 W$ and $Z$ decays
10.5 Experimental results
10.6 Constraints on new physics

\subsection{Introduction}

The standard electroweak model is based on the gauge group [1] $\mathrm{SU}(2) \times \mathrm{U}(1)$, with gauge bosons $W_{\mu}^{i}, i=1,2,3$, and $B_{\mu}$ for the $\mathrm{SU}(2)$ and $\mathrm{U}(1)$ factors, respectively, and the corresponding gauge coupling constants $g$ and $g^{\prime}$. The left-handed fermion fields

$\psi_{i}=\left(\begin{array}{c}\nu_{i} \\ \ell_{i}^{-}\end{array}\right)$and $\left(\begin{array}{c}u_{i} \\ d_{i}^{\prime}\end{array}\right)$ of the $i^{\text {th }}$ fermion family transform as doublets under $\mathrm{SU}(2)$, where $d_{i}^{\prime} \equiv \sum_{j} V_{i j} d_{j}$, and $V$ is the Cabibbo-Kobayashi-Maskawa mixing matrix. (Constraints on $V$ are discussed in the section on the Cabibbo-Kobayashi-Maskawa mixing matrix.) The right-handed fields are $\mathrm{SU}(2)$ singlets. In the minimal model there are three fermion families and a single complex Higgs doublet $\phi \equiv\left(\begin{array}{c}\phi^{+} \\ \phi^{0}\end{array}\right)$.

After spontaneous symmetry breaking the Lagrangian for the fermion fields is

$$
\begin{aligned}
\mathscr{L}_{F} & =\sum_{i} \bar{\psi}_{i}\left(i \not \partial-m_{i}-\frac{g m_{i} H}{2 M_{W}}\right) \psi_{i} \\
& -\frac{g}{2 \sqrt{2}} \sum_{i} \bar{\psi}_{i} \gamma^{\mu}\left(1-\gamma^{5}\right)\left(T^{+} W_{\mu}^{+}+T^{-} W_{\mu}^{-}\right) \psi_{i} \\
& -e \sum_{i} q_{i} \bar{\psi}_{i} \gamma^{\mu} \psi_{i} A_{\mu} \\
& -\frac{g}{2 \cos \theta_{W}} \sum_{i} \bar{\psi}_{i} \gamma^{\mu}\left(g_{V}^{i}-g_{A}^{i} \gamma^{5}\right) \psi_{i} Z_{\mu} .
\end{aligned}
$$

$\theta_{W} \equiv \tan ^{-1}\left(g^{\prime} / g\right)$ is the weak angle; $e=g \sin \theta_{W}$ is the positron electric charge; and $A \equiv B \cos \theta_{W}+W^{3} \sin \theta_{W}$ is the (massless) photon field. $W^{ \pm} \equiv\left(W^{1} \mp i W^{2}\right) / \sqrt{2}$ and $Z \equiv-B \sin \theta_{W}+W^{3} \cos \theta_{W}$ are the massive charged and neutral weak boson fields, respectively. $T^{+}$and $T^{-}$are the weak isospin raising and lowering operators. The vector and axial couplings are

$$
\begin{aligned}
g_{V}^{i} & \equiv t_{3 L}(i)-2 q_{i} \sin ^{2} \theta_{W}, \\
g_{A}^{i} & \equiv t_{3 L}(i),
\end{aligned}
$$




\section{10. Electroweak model and constraints on new physics}

where $t_{3 L}(i)$ is the weak isospin of fermion $i\left(+1 / 2\right.$ for $u_{i}$ and $\nu_{i} ;-1 / 2$ for $d_{i}$ and $\left.e_{i}\right)$ and $q_{i}$ is the charge of $\psi_{i}$ in units of $e$.

The second term in $\mathscr{L}_{F}$ represents the charged-current weak interaction [2]. For example, the coupling of a $W$ to an electron and a neutrino is

$$
-\frac{e}{2 \sqrt{2} \sin \theta_{W}}\left[W_{\mu}^{-} \bar{e} \gamma^{\mu}\left(1-\gamma^{5}\right) \nu+W_{\mu}^{+} \bar{\nu} \gamma^{\mu}\left(1-\gamma^{5}\right) e\right] .
$$

For momenta small compared to $M_{W}$, this term gives rise to the effective four-fermion interaction with the Fermi constant given (at tree level, i.e., lowest order in perturbation theory) by $G_{F} / \sqrt{2}=g^{2} / 8 M_{W}^{2}$. $C P$ violation is incorporated in the Standard Model by a single observable phase in $V_{i j}$. The third term in $\mathscr{L}_{F}$ describes electromagnetic interactions (QED), and the last is the weak neutral-current interaction.

In Eq. (10.1), $m_{i}$ is the mass of the $i^{\text {th }}$ fermion $\psi_{i}$. For the quarks these are the current masses. For the light quarks, as described in the Particle Listings, $\bar{m}_{u} \approx 2-8 \mathrm{MeV}$, $\bar{m}_{d} \approx 5-15 \mathrm{MeV}$, and $\bar{m}_{s} \approx 100-300 \mathrm{MeV}$ (these are running $\overline{\mathrm{MS}}$ masses evaluated at $\mu=1 \mathrm{GeV})$. For the heavier quarks, the $\overline{\mathrm{MS}}$ masses are $\bar{m}_{c}\left(\mu=\bar{m}_{c}\right) \approx 1.0-1.6 \mathrm{GeV}$ and $\bar{m}_{b}\left(\mu=\bar{m}_{b}\right) \approx 4.1-4.5 \mathrm{GeV}$. The average of the recent CDF [4] and DØ [5] values for the top quark "pole" mass is $m_{t}=175 \pm 5 \mathrm{GeV}$. See "The Note on Quark Masses" in the Particle Listings for more information.

$H$ is the physical neutral Higgs scalar which is the only remaining part of $\phi$ after spontaneous symmetry breaking. The Yukawa coupling of $H$ to $\psi_{i}$, which is flavor diagonal in the minimal model, is $g m_{i} / 2 M_{W}$. The $H$ mass is not predicted by the model. Experimental limits are given in the Higgs section. In nonminimal models there are additional charged and neutral scalar Higgs particles [6].

\subsection{Renormalization and radiative corrections}

The Standard Model has three parameters (not counting $M_{H}$ and the fermion masses and mixings). A particularly useful set is:

(a) The fine structure constant $\alpha=1 / 137.0359895$ (61), determined from the quantum Hall effect. In most electroweak-renormalization schemes, it is convenient to define a running $\alpha$ dependent on the energy scale of the process, with $\alpha^{-1} \sim 137$ appropriate at low energy. (The running has recently been observed directly [7].) At energies of order $M_{Z}, \alpha^{-1} \sim 128$. For example, in the modified minimal subtraction $(\overline{\mathrm{MS}})$ scheme [8], one has $\widehat{\alpha}\left(M_{Z}\right)^{-1}=127.88 \pm 0.09$, while the conventional (on-shell) QED renormalization yields [9] $\alpha\left(M_{Z}\right)^{-1}=128.88 \pm 0.09$, which differs by finite constants from $\widehat{\alpha}\left(M_{Z}\right)^{-1}$. The uncertainty, due to the low-energy hadronic contribution to vacuum polarization, is the dominant theoretical uncertainty in the interpretation of precision data. Other recent evaluations [10-14] of this effect are in reasonable agreement. Further improvement will require better measurements of the cross section for $e^{+} e^{-} \rightarrow$ hadrons at low energy.

(b) The Fermi constant, $G_{F}=1.16639(1) \times 10^{-5} \mathrm{GeV}^{-2}$, determined from the muon lifetime formula [15],

$$
\tau_{\mu}^{-1}=\frac{G_{F}^{2} m_{\mu}^{5}}{192 \pi^{3}} F\left(\frac{m_{e}^{2}}{m_{\mu}^{2}}\right)\left(1+\frac{3}{5} \frac{m_{\mu}^{2}}{M_{W}^{2}}\right)
$$




$$
\times\left[1+\frac{\alpha\left(m_{\mu}\right)}{2 \pi}\left(\frac{25}{4}-\pi^{2}\right)\right],
$$

where

$$
F(x)=1-8 x+8 x^{3}-x^{4}-12 x^{2} \ln x,
$$

and

$$
\alpha\left(m_{\mu}\right)^{-1}=\alpha^{-1}-\frac{2}{3 \pi} \ln \left(\frac{m_{\mu}}{m_{e}}\right)+\frac{1}{6 \pi} \approx 136
$$

where the uncertainty in $G_{F}$ is from the input quantities. There are additional uncertainties from higher order radiative corrections, which can be estimated from the magnitude of the known $\alpha^{2} \ln \left(m_{\mu} / m_{e}\right)$ term of $\sim 1.8 \times 10^{-10}$ (alternatively, one can view Eq. (10.5) as the exact definition of $G_{F}$; then the theoretical uncertainty appears instead in the formulae for quantities derived from $G_{F}$ ).

(c) $\sin ^{2} \theta_{W}$, determined from the $Z$ mass and other $Z$ pole observables, the $W$ mass, and neutral-current processes [16]. The value of $\sin ^{2} \theta_{W}$ depends on the renormalization prescription. There are a number of popular schemes [18-23] leading to $\sin ^{2} \theta_{W}$ values which differ by small factors which depend on $m_{t}$ and $M_{H}$. The notation for these schemes is shown in Table 10.1. Discussion of the schemes follows the table.

Table 10.1: Notations used to indicate the various schemes discussed in the text. Each definition of $\sin \theta_{W}$ leads to values that differ by small factors depending on $m_{t}$ and $M_{H}$.

\begin{tabular}{ll}
\hline \hline Scheme & \multicolumn{1}{c}{ Notation } \\
\hline On-shell & $s_{W}=\sin \theta_{W}$ \\
NOV & $s_{M_{Z}}=\sin \theta_{W}$ \\
$\overline{\mathrm{MS}}$ & $\widehat{s}_{Z}=\sin \theta_{W}$ \\
$\overline{\mathrm{MS}} N D$ & $\widehat{s}_{N D}=\sin \theta_{W}$ \\
Effective angle & $\bar{s}_{f}=\sin \theta_{W}$ \\
\hline \hline
\end{tabular}

(i) The on-shell scheme promotes the tree-level formula $\sin ^{2} \theta_{W}=1-M_{W}^{2} / M_{Z}^{2}$ to a definition of the renormalized $\sin ^{2} \theta_{W}$ to all orders in perturbation theory, i.e., $\sin ^{2} \theta_{W} \rightarrow s_{W}^{2} \equiv 1-M_{W}^{2} / M_{Z}^{2}$. This scheme is simple conceptually. However, $M_{W}$ is known much less precisely than $M_{Z}$ and in practice one extracts $s_{W}^{2}$ from $M_{Z}$ alone using

$$
\begin{aligned}
M_{W} & =\frac{A_{0}}{s_{W}(1-\Delta r)^{1 / 2}}, \\
M_{Z} & =\frac{M_{W}}{c_{W}}
\end{aligned}
$$




\section{10. Electroweak model and constraints on new physics}

where $s_{W} \equiv \sin \theta_{W}, c_{W} \equiv \cos \theta_{W}, A_{0}=\left(\pi \alpha / \sqrt{2} G_{F}\right)^{1 / 2}=37.2802 \mathrm{GeV}$, and $\Delta r$ includes the radiative corrections relating $\alpha, \alpha\left(M_{Z}\right), G_{F}, M_{W}$, and $M_{Z}$. One finds $\Delta r \sim \Delta r_{0}-\rho_{t} / \tan ^{2} \theta_{W}$, where $\Delta r_{0} \approx 1-\alpha / \alpha\left(M_{Z}\right) \approx 0.06$ is due to the running of $\alpha$ and $\rho_{t}=3 G_{F} m_{t}^{2} / 8 \sqrt{2} \pi^{2} \approx 0.0096\left(m_{t} / 175 \mathrm{GeV}\right)^{2}$ represents the dominant (quadratic) $m_{t}$ dependence. There are additional contributions to $\Delta r$ from bosonic loops, including those which depend logarithmically on the Higgs mass $M_{H}$. One has $\Delta r=0.0349 \mp 0.0019 \pm 0.0007$ for $\left(m_{t}, M_{H}\right)=\left(175 \pm 5 \mathrm{GeV}, M_{Z}\right)$, where the second uncertainty is from $\alpha\left(M_{Z}\right)$. Thus the value of $s_{W}^{2}$ extracted from $M_{Z}$ includes a large uncertainty $(\mp 0.0006)$ from the currently allowed range of $m_{t}$.

(ii) A more precisely determined quantity $s_{M_{Z}}^{2}$ can be obtained from $M_{Z}$ by removing the $\left(m_{t}, M_{H}\right)$ dependent term from $\Delta r[19]$, i.e.,

$$
s_{M_{Z}}^{2} c_{M_{Z}}^{2} \equiv \frac{\pi \alpha\left(M_{Z}\right)}{\sqrt{2} G_{F} M_{Z}^{2}} .
$$

This yields $s_{M_{Z}}^{2}=0.23116 \pm 0.00022$, with most of the uncertainty from $\alpha$ rather than $M_{Z}$. Scheme $(i i)$ is equivalent to using $M_{Z}$ rather than $\sin ^{2} \theta_{W}$ as the third fundamental parameter. However, it recognizes that $s_{M_{Z}}^{2}$ is still a useful derived quantity. The small uncertainty in $s_{M_{Z}}^{2}$ compared to other schemes is because the $m_{t}$ dependence has been removed by definition. However, the $m_{t}$ uncertainty reemerges when other quantities $\left(e . g ., M_{W}\right.$ or other $Z$ pole observables) are predicted in terms of $M_{Z}$.

Both $s_{W}^{2}$ and $s_{M_{Z}}^{2}$ depend not only on the gauge couplings but also on the spontaneous-symmetry breaking, and both definitions are awkward in the presence of any extension of the Standard Model which perturbs the value of $M_{Z}$ (or $M_{W}$ ). Other definitions are motivated by the tree-level coupling constant definition $\theta_{W}=\tan ^{-1}\left(g^{\prime} / g\right)$.

(iii) In particular, the modified minimal subtraction $(\overline{\mathrm{MS}})$ scheme introduces the quantity $\sin ^{2} \widehat{\theta}_{W}(\mu) \equiv \widehat{g}^{\prime 2}(\mu) /\left[\widehat{g}^{2}(\mu)+\widehat{g}^{\prime 2}(\mu)\right]$, where the couplings $\widehat{g}$ and $\widehat{g}^{\prime}$ are defined by modified minimal subtraction and the scale $\mu$ is conveniently chosen to be $M_{Z}$ for electroweak processes. The value of $\widehat{s}_{Z}^{2}=\sin ^{2} \widehat{\theta}_{W}\left(M_{Z}\right)$ extracted from $M_{Z}$ is less sensitive than $s_{W}^{2}$ to $m_{t}$ (by a factor of $\tan ^{2} \theta_{W}$ ), and is less sensitive to most types of new physics than $s_{W}^{2}$ or $s_{M_{Z}}^{2}$. It is also very useful for comparing with the predictions of grand unification. There are actually several variant definitions of $\sin ^{2} \widehat{\theta}_{W}\left(M_{Z}\right)$, differing according to whether or how finite $\alpha \ln \left(m_{t} / M_{Z}\right)$ terms are decoupled (subtracted from the couplings). One cannot entirely decouple the $\alpha \ln \left(m_{t} / M_{Z}\right)$ terms from all electroweak quantities because $m_{t} \gg m_{b}$ breaks $\mathrm{SU}(2)$ symmetry. The scheme that will be adopted here decouples the $\alpha \ln \left(m_{t} / M_{Z}\right)$ terms from the $\gamma-Z$ mixing [8,20], essentially eliminating any $\ln \left(m_{t} / M_{Z}\right)$ dependence in the formulae for asymmetries at the $Z$ pole when written in terms of $\widehat{s}_{Z}^{2}$. The various definitions are related by

$$
\widehat{s}_{Z}^{2}=c\left(m_{t}, M_{H}\right) s_{W}^{2}=\bar{c}\left(m_{t}, M_{H}\right) s_{M_{Z}}^{2},
$$


where $c=1.0376 \pm 0.0021$ for $m_{t}=175 \pm 5 \mathrm{GeV}$ and $M_{H}=M_{Z}$. Similarly, $\bar{c}=1.0003 \mp 0.0007$. The quadratic $m_{t}$ dependence is given by $c \sim 1+\rho_{t} / \tan ^{2} \theta_{W}$ and $\bar{c} \sim 1-\rho_{t} /\left(1-\tan ^{2} \theta_{W}\right)$, respectively. The expressions for $M_{W}$ and $M_{Z}$ in the $\overline{\mathrm{MS}}$ scheme are

$$
\begin{aligned}
M_{W} & =\frac{A_{0}}{\widehat{s}_{Z}\left(1-\Delta \widehat{r}_{W}\right)^{1 / 2}}, \\
M_{Z} & =\frac{M_{W}}{\widehat{\rho}^{1 / 2} \widehat{c}_{Z}} .
\end{aligned}
$$

One predicts $\Delta \widehat{r}_{W}=0.0698 \pm 0.0001 \pm 0.0007$ for $m_{t}=175 \pm 5 \mathrm{GeV}$ and $M_{H}=M_{Z} \cdot \Delta \widehat{r}_{W}$ has no quadratic $m_{t}$ dependence, because shifts in $M_{W}$ are absorbed into the observed $G_{F}$, so that the error in $\Delta \widehat{r}_{W}$ is dominated by $\Delta r_{0}=1-\alpha / \alpha\left(M_{Z}\right)$, which induces the second quoted uncertainty. Similarly, $\widehat{\rho} \sim 1+\rho_{t}$. Including bosonic loops, $\widehat{\rho}=1.0109 \pm 0.0006$ for $\left(m_{t}, M_{H}\right)=\left(175 \pm 5 \mathrm{GeV}, M_{Z}\right)$.

(iv) A variant $\overline{\mathrm{MS}}$ quantity $\widehat{s}_{\mathrm{ND}}^{2}$ (used in the 1992 edition of this Review) does not decouple the $\alpha \ln \left(m_{t} / M_{Z}\right)$ terms [21]. It is related to $\widehat{s}_{Z}^{2}$ by

$$
\begin{aligned}
\widehat{s}_{Z}^{2} & =\widehat{s}_{\mathrm{ND}}^{2} /\left(1+\frac{\widehat{\alpha}}{\pi} d\right), \\
d & =\frac{1}{3}\left(\frac{1}{\widehat{s}^{2}}-\frac{8}{3}\right)\left[\left(1+\frac{\widehat{\alpha}_{s}}{\pi}\right) \ln \frac{m_{t}}{M_{Z}}-\frac{15 \widehat{\alpha}_{s}}{8 \pi}\right],
\end{aligned}
$$

where $\widehat{\alpha}_{s}$ is the QCD coupling at $M_{Z}$. Thus, $\widehat{s}_{Z}^{2}-\widehat{s}_{\mathrm{ND}}^{2} \sim-0.0002$ for $m_{t}=175 \mathrm{GeV}$.

$(v)$ Yet another definition, the effective angle $[22,23] \bar{s}_{f}^{2}$ for $Z$ coupling to fermion $f$, is described at the end of Sec. 10.3.

Experiments are now at such a level of precision that complete $\mathcal{O}(\alpha)$ radiative corrections must be applied. For neutral-current and $Z$ pole processes, these corrections are conveniently divided into two classes:

1. QED diagrams involving the emission of real photons or the exchange of virtual photons in loops, but not including vacuum polarization diagrams. These graphs often yield finite and gauge-invariant contributions to observable processes. However, they are dependent on energies, experimental cuts, etc., and must be calculated individually for each experiment.

2. Electroweak corrections, including $\gamma \gamma, \gamma Z, Z Z$, and $W W$ vacuum polarization diagrams, as well as vertex corrections, box graphs, etc., involving virtual $W$ 's and Z's. Many of these corrections are absorbed into the renormalized Fermi constant defined in Eq. (10.5). Others modify the tree-level expressions for $Z$ pole observables and neutral-current amplitudes in several ways [16]. One-loop corrections are included for all processes. In addition, certain two-loop corrections are also important. In particular, two-loop corrections involving the top-quark modify $\rho_{t}$ in $\widehat{\rho}, \Delta r$, and elsewhere by

$$
\rho_{t} \rightarrow \rho_{t}\left[1+R\left(M_{H}, m_{t}\right) \rho_{t} / 3\right]
$$




\section{10. Electroweak model and constraints on new physics}

$R\left(M_{H}, m_{t}\right)$ is best described as an expansion in $M_{Z}^{2} / m_{t}^{2}$. The unsuppressed terms were first obtained in Ref. 24, and are known analytically [25]. Contributions proportional to $M_{Z}^{2} / m_{t}^{2}$ were studied in Ref. 26 with the help of small and large Higgs mass expansions, which can be interpolated. These contributions are about as large as the leading ones in Refs. 24 and 25. Very recently, a subset of the relevant two-loop diagrams has been calculated numerically without any heavy mass expansion [27]. This serves as a valuable check on the $M_{H}$ dependence of the leading terms obtained in Refs. 24-26. The difference turned out to be small. For $M_{H}$ above its lower direct limit, $-17<R<-11$. Mixed QCD-electroweak loops of order $\alpha \alpha_{s} m_{t}^{2}$ [28] and $\alpha \alpha_{s}^{2} m_{t}^{2}$ [29] increase the predicted value of $m_{t}$ by $6 \%$. This is, however, almost entirely an artifact of using the pole mass definition for $m_{t}$. The equivalent corrections when using the $\overline{\mathrm{MS}}$ definition $\bar{m}_{t}\left(\bar{m}_{t}\right)$ increase $m_{t}$ by less than $0.5 \%$. The leading electroweak $[24,25]$ and mixed [30] two-loop terms are also known for the $Z \rightarrow b \bar{b}$ vertex, but not the respective subleading ones.

\subsection{Cross-section and asymmetry formulas}

It is convenient to write the four-fermion interactions relevant to $\nu$-hadron, $\nu e$, and parity violating $e$-hadron neutral-current processes in a form that is valid in an arbitrary gauge theory (assuming massless left-handed neutrinos). One has

$$
\begin{gathered}
-\mathscr{L}^{\nu \text { Hadron }}=\frac{G_{F}}{\sqrt{2}} \bar{\nu} \gamma^{\mu}\left(1-\gamma^{5}\right) \nu \\
\times \sum_{i}\left[\epsilon_{L}(i) \bar{q}_{i} \gamma_{\mu}\left(1-\gamma^{5}\right) q_{i}+\epsilon_{R}(i) \bar{q}_{i} \gamma_{\mu}\left(1+\gamma^{5}\right) q_{i}\right], \\
-\mathscr{L}^{\nu e}=\frac{G_{F}}{\sqrt{2}} \bar{\nu}_{\mu} \gamma^{\mu}\left(1-\gamma^{5}\right) \nu_{\mu} \bar{e} \gamma_{\mu}\left(g_{V}^{\nu e}-g_{A}^{\nu e} \gamma^{5}\right) e
\end{gathered}
$$

(for $\nu_{e} e$ or $\bar{\nu}_{e} e$, the charged-current contribution must be included), and

$$
\begin{gathered}
-\mathscr{L}^{e \text { Hadron }}=-\frac{G_{F}}{\sqrt{2}} \\
\times \sum_{i}\left[C_{1 i} \bar{e} \gamma_{\mu} \gamma^{5} e \bar{q}_{i} \gamma^{\mu} q_{i}+C_{2 i} \bar{e} \gamma_{\mu} e \bar{q}_{i} \gamma^{\mu} \gamma^{5} q_{i}\right] .
\end{gathered}
$$

(One must add the parity-conserving QED contribution.)

The Standard Model expressions for $\epsilon_{L, R}(i), g_{V, A}^{\nu e}$, and $C_{i j}$ are given in Table 10.2. Note that $g_{V, A}^{\nu e}$ and the other quantities are coefficients of effective four-fermi operators, which differ from the quantities defined in Eq. (10.2) and Eq. (10.3) in the radiative corrections and in the presence of possible physics beyond the Standard Model.

A precise determination of the on-shell $s_{W}^{2}$, which depends only very weakly on $m_{t}$ and $M_{H}$, is obtained from deep inelastic neutrino scattering from (approximately) isoscalar 
targets [31]. The ratio $R_{\nu} \equiv \sigma_{\nu N}^{N C} / \sigma_{\nu N}^{C C}$ of neutral- to charged-current cross sections has been measured to $1 \%$ accuracy by the CDHS [32] and CHARM [33] collaborations at CERN [34], and the CCFR collaboration at Fermilab [35,36] has obtained an even more precise result, so it is important to obtain theoretical expressions for $R_{\nu}$ and $R_{\bar{\nu}} \equiv \sigma_{\bar{\nu} N}^{N C} / \sigma_{\bar{\nu} N}^{C C}$ (as functions of $\sin ^{2} \theta_{W}$ ) to comparable accuracy. Fortunately, most of the uncertainties from the strong interactions and neutrino spectra cancel in the ratio.

Table 10.2: Standard Model expressions for the neutral-current parameters for $\nu$-hadron, $\nu e$, and $e$-hadron processes. At tree level, $\rho=\kappa=1, \lambda=0$. If radiative corrections are included, $\rho_{\nu N}^{N C}=1.0084, \widehat{\kappa}_{\nu N}=0.9964\left(\right.$ at $\left.\left\langle Q^{2}\right\rangle=35 \mathrm{GeV}^{2}\right)$, $\lambda_{u L}=-0.0031, \lambda_{d L}=-0.0025$, and $\lambda_{d R}=2 \lambda_{u R}=7.5 \times 10^{-5}$ for $m_{t}=175 \mathrm{GeV}$ and $M_{H}=M_{Z}=91.1867 \mathrm{GeV}$. For $\nu e$ scattering, $\rho_{\nu e}=1.0130$ and $\widehat{\kappa}_{\nu e}=0.9970$ (at $\left\langle Q^{2}\right\rangle=0$.). For atomic parity violation and the SLAC polarized electron experiment, $\rho_{e q}^{\prime}=0.9879, \rho_{e q}=1.0009, \widehat{\kappa}_{e q}^{\prime}=1.0029, \widehat{\kappa}_{e q}=1.0304, \lambda_{1 d}=-2 \lambda_{1 u}=3.7 \times 10^{-5}$, $\lambda_{2 u}=-0.0121$ and $\lambda_{2 d}=0.0026$. The dominant $m_{t}$ dependence is given by $\rho \sim 1+\rho_{t}$, while $\widehat{\kappa} \sim 1(\overline{\mathrm{MS}})$ or $\kappa \sim 1+\rho_{t} / \tan ^{2} \theta_{W}$ (on-shell).

\begin{tabular}{ll}
\hline \hline Quantity & Standard Model Expression \\
\hline$\epsilon_{L}(u)$ & $\rho_{\nu N}^{N C}\left(\frac{1}{2}-\frac{2}{3} \widehat{\kappa}_{\nu N} \widehat{s}_{Z}^{2}\right)+\lambda_{u L}$ \\
$\epsilon_{L}(d)$ & $\rho_{\nu N}^{N C}\left(-\frac{1}{2}+\frac{1}{3} \widehat{\kappa}_{\nu N} \widehat{s}_{Z}^{2}\right)+\lambda_{d L}$ \\
$\epsilon_{R}(u)$ & $\rho_{\nu N}^{N C}\left(-\frac{2}{3} \widehat{\kappa}_{\nu N} \widehat{s}_{Z}^{2}\right)+\lambda_{u R}$ \\
$\epsilon_{R}(d)$ & $\rho_{\nu N}^{N C}\left(\frac{1}{3} \widehat{\kappa}_{\nu N} \widehat{s}_{Z}^{2}\right)+\lambda_{d R}$ \\
$g_{V}^{\nu e}$ & $\rho_{\nu e}\left(-\frac{1}{2}+2 \widehat{\kappa}_{\nu e} \widehat{s}_{Z}^{2}\right)$ \\
$g_{A}^{\nu e}$ & $\rho_{\nu e}\left(-\frac{1}{2}\right)$ \\
$C_{1 u}$ & $\rho_{e q}^{\prime}\left(-\frac{1}{2}+\frac{4}{3} \widehat{\kappa}_{e q}^{\prime} \widehat{s}_{Z}^{2}\right)+\lambda_{1 u}$ \\
$C_{1 d}$ & $\rho_{e q}^{\prime}\left(\frac{1}{2}-\frac{2}{3} \widehat{\kappa}_{e q}^{\prime} \widehat{s}_{Z}^{2}\right)+\lambda_{1 d}$ \\
$C_{2 u}$ & $\rho_{e q}\left(-\frac{1}{2}+2 \widehat{\kappa}_{e q} \widehat{s}_{Z}^{2}\right)+\lambda_{2 u}$ \\
$C_{2 d}$ & $\rho_{e q}\left(\frac{1}{2}-2 \widehat{\kappa}_{e q} \widehat{s}_{Z}^{2}\right)+\lambda_{2 d}$ \\
\hline \hline
\end{tabular}

A simple zero $^{\text {th }}$-order approximation is

$$
\begin{aligned}
& R_{\nu}=g_{L}^{2}+g_{R}^{2} r \\
& R_{\bar{\nu}}=g_{L}^{2}+\frac{g_{R}^{2}}{r}
\end{aligned}
$$




\section{10. Electroweak model and constraints on new physics}

where

$$
\begin{gathered}
g_{L}^{2} \equiv \epsilon_{L}(u)^{2}+\epsilon_{L}(d)^{2} \approx \frac{1}{2}-\sin ^{2} \theta_{W}+\frac{5}{9} \sin ^{4} \theta_{W}, \\
g_{R}^{2} \equiv \epsilon_{R}(u)^{2}+\epsilon_{R}(d)^{2} \approx \frac{5}{9} \sin ^{4} \theta_{W},
\end{gathered}
$$

and $r \equiv \sigma_{\bar{\nu} N}^{C C} / \sigma_{\nu N}^{C C}$ is the ratio of $\bar{\nu}$ and $\nu$ charged-current cross sections, which can be measured directly. (In the simple parton model, ignoring hadron energy cuts, $r \approx\left(\frac{1}{3}+\epsilon\right) /\left(1+\frac{1}{3} \epsilon\right)$, where $\epsilon \sim 0.125$ is the ratio of the fraction of the nucleon's momentum carried by antiquarks to that carried by quarks.) In practice, Eq. (10.15) must be corrected for quark mixing, quark sea effects, $c$-quark threshold effects, nonisoscalarity, $W-Z$ propagator differences, the finite muon mass, QED and electroweak radiative corrections. Details of the neutrino spectra, experimental cuts, $x$ and $Q^{2}$ dependence of structure functions, and longitudinal structure functions enter only at the level of these corrections and therefore lead to very small uncertainties. The largest theoretical uncertainty is associated with the $c$-threshold, which mainly affects $\sigma^{C C}$. Using the slow rescaling prescription [37] the central value of $\sin ^{2} \theta_{W}$ from CCFR varies as $0.0111\left(m_{c}[\mathrm{GeV}]-1.31\right)$, where $m_{c}$ is the effective mass. For $m_{c}=1.31 \pm 0.24 \mathrm{GeV}$ (determined from $\nu$-induced dimuon production [38]) this contributes \pm 0.003 to the total uncertainty $\Delta \sin ^{2} \theta_{W} \sim \pm 0.004$. This would require a high-energy neutrino beam for improvement. (The experimental uncertainty is also \pm 0.003 ). The CCFR group quotes $s_{W}^{2}=0.2236 \pm 0.0041$ for $\left(m_{t}, M_{H}\right)=(175,150) \mathrm{GeV}$ with very little sensitivity to $\left(m_{t}, M_{H}\right)$. Combining all of the precise deep-inelastic measurements, one obtains $s_{W}^{2}=0.2260 \pm 0.0039$.

The laboratory cross section for $\nu_{\mu} e \rightarrow \nu_{\mu} e$ or $\bar{\nu}_{\mu} e \rightarrow \bar{\nu}_{\mu} e$ elastic scattering is

$$
\begin{gathered}
\frac{d \sigma_{\nu_{\mu}, \bar{\nu} \mu}}{d y}=\frac{G_{F}^{2} m_{e} E_{\nu}}{2 \pi} \\
\times\left[\left(g_{V}^{\nu e} \pm g_{A}^{\nu e}\right)^{2}+\left(g_{V}^{\nu e} \mp g_{A}^{\nu e}\right)^{2}(1-y)^{2}\right. \\
\left.-\left(g_{V}^{\nu e 2}-g_{A}^{\nu e 2}\right) \frac{y m_{e}}{E_{\nu}}\right],
\end{gathered}
$$

where the upper (lower) sign refers to $\nu_{\mu}\left(\bar{\nu}_{\mu}\right)$, and $y \equiv E_{e} / E_{\nu}$ (which runs from 0 to $\left.\left(1+m_{e} / 2 E_{\nu}\right)^{-1}\right)$ is the ratio of the kinetic energy of the recoil electron to the incident $\nu$ or $\bar{\nu}$ energy. For $E_{\nu} \gg m_{e}$ this yields a total cross section

$$
\sigma=\frac{G_{F}^{2} m_{e} E_{\nu}}{2 \pi}\left[\left(g_{V}^{\nu e} \pm g_{A}^{\nu e}\right)^{2}+\frac{1}{3}\left(g_{V}^{\nu e} \mp g_{A}^{\nu e}\right)^{2}\right] \text {. }
$$

The most accurate leptonic measurements [39-41] of $\sin ^{2} \theta_{W}$ are from the ratio $R \equiv \sigma_{\nu_{\mu} e} / \sigma_{\bar{\nu} \mu}$ in which many of the systematic uncertainties cancel. Radiative corrections (other than $m_{t}$ effects) are small compared to the precision of present experiments and have negligible effect on the extracted $\sin ^{2} \theta_{W}$. The most precise 
experiment (CHARM II) [41] determined not only $\sin ^{2} \theta_{W}$ but $g_{V, A}^{\nu e}$ as well. The cross sections for $\nu_{e} e$ and $\bar{\nu}_{e} e$ may be obtained from Eq. (10.17) by replacing $g_{V, A}^{\nu e}$ by $g_{V, A}^{\nu e}+1$, where the 1 is due to the charged-current contribution.

The SLAC polarized-electron experiment [42] measured the parity-violating asymmetry

$$
A=\frac{\sigma_{R}-\sigma_{L}}{\sigma_{R}+\sigma_{L}},
$$

where $\sigma_{R, L}$ is the cross section for the deep-inelastic scattering of a right- or left-handed electron: $e_{R, L} N \rightarrow e \mathrm{X}$. In the quark parton model

$$
\frac{A}{Q^{2}}=a_{1}+a_{2} \frac{1-(1-y)^{2}}{1+(1-y)^{2}}
$$

where $Q^{2}>0$ is the momentum transfer and $y$ is the fractional energy transfer from the electron to the hadrons. For the deuteron or other isoscalar targets, one has, neglecting the $s$-quark and antiquarks,

$$
\begin{gathered}
a_{1}=\frac{3 G_{F}}{5 \sqrt{2} \pi \alpha}\left(C_{1 u}-\frac{1}{2} C_{1 d}\right) \approx \frac{3 G_{F}}{5 \sqrt{2} \pi \alpha}\left(-\frac{3}{4}+\frac{5}{3} \sin ^{2} \theta_{W}\right), \\
a_{2}=\frac{3 G_{F}}{5 \sqrt{2} \pi \alpha}\left(C_{2 u}-\frac{1}{2} C_{2 d}\right) \approx \frac{9 G_{F}}{5 \sqrt{2} \pi \alpha}\left(\sin ^{2} \theta_{W}-\frac{1}{4}\right) .
\end{gathered}
$$

There are now precise experiments measuring atomic parity violation [43] in cesium (at the $0.4 \%$ level) [44], thallium [45], lead [46], and bismuth [47]. The uncertainties associated with atomic wave functions are quite small for cesium, for which they are $\sim 1 \%$ [48]. The theoretical uncertainties are 3\% for thallium [49] but larger for the other atoms. For heavy atoms one determines the "weak charge"

$$
\begin{gathered}
Q_{W}=-2\left[C_{1 u}(2 Z+N)+C_{1 d}(Z+2 N)\right] \\
\approx Z\left(1-4 \sin ^{2} \theta_{W}\right)-N .
\end{gathered}
$$

The recent Boulder experiment in cesium also observed the parity-violating weak corrections to the nuclear electromagnetic vertex (the anapole moment [50]).

In the future it should be possible to reduce the theoretical wave function uncertainties by taking the ratios of parity violation in different isotopes [43,51]. There would still be some residual uncertainties from differences in the neutron charge radii, however [52].

The forward-backward asymmetry for $e^{+} e^{-} \rightarrow \ell^{+} \ell^{-}, \ell=\mu$ or $\tau$, is defined as

$$
A_{F B} \equiv \frac{\sigma_{F}-\sigma_{B}}{\sigma_{F}+\sigma_{B}}
$$

where $\sigma_{F}\left(\sigma_{B}\right)$ is the cross section for $\ell^{-}$to travel forward (backward) with respect to the $e^{-}$direction. $A_{F B}$ and $R$, the total cross section relative to pure QED, are given by

$$
R=F_{1}
$$




\section{10. Electroweak model and constraints on new physics}

$$
A_{F B}=3 F_{2} / 4 F_{1},
$$

where

$$
\begin{gathered}
F_{1}=1-2 \chi_{0} g_{V}^{e} g_{V}^{\ell} \cos \delta_{R}+\chi_{0}^{2}\left(g_{V}^{e 2}+g_{A}^{e 2}\right)\left(g_{V}^{\ell 2}+g_{A}^{\ell 2}\right), \\
F_{2}=-2 \chi_{0} g_{A}^{e} g_{A}^{\ell} \cos \delta_{R}+4 \chi_{0}^{2} g_{A}^{e} g_{A}^{\ell} g_{V}^{e} g_{V}^{\ell}, \\
\tan \delta_{R}=\frac{M_{Z} \Gamma_{Z}}{M_{Z}^{2}-s} \\
\chi_{0}=\frac{G_{F}}{2 \sqrt{2} \pi \alpha} \frac{s M_{Z}^{2}}{\left[\left(M_{Z}^{2}-s\right)^{2}+M_{Z}^{2} \Gamma_{Z}^{2}\right]^{1 / 2}},
\end{gathered}
$$

and $\sqrt{s}$ is the CM energy. Eq. (10.26) is valid at tree level. If the data is radiatively corrected for QED effects (as described above), then the remaining electroweak corrections can be incorporated [53,54] (in an approximation adequate for existing PEP, PETRA, and TRISTAN data, which are well below the $Z$ pole) by replacing $\chi_{0}$ by $\chi(s) \equiv\left(1+\rho_{t}\right) \chi_{0}(s) \alpha / \alpha(s)$, where $\alpha(s)$ is the running QED coupling, and evaluating $g_{V}$ in the $\overline{\mathrm{MS}}$ scheme. Formulas for $e^{+} e^{-} \rightarrow$ hadrons may be found in Ref. 55 .

At LEP and SLC, there are high-precision measurements of various $Z$ pole observables [56-61]. These include the $Z$ mass and total width, $\Gamma_{Z}$, and partial widths $\Gamma(f \bar{f})$ for $Z \rightarrow f \bar{f}$ where fermion $f=e, \mu, \tau$, hadrons, $b$, or $c$. The data is consistent with lepton-family universality, $\Gamma\left(e^{+} e^{-}\right)=\Gamma\left(\mu^{+} \mu^{-}\right)=\Gamma\left(\tau^{+} \tau^{-}\right)$, so one may work with an average width $\Gamma\left(\ell^{+} \ell^{-}\right)$. It is convenient to use the variables $M_{Z}$, $\Gamma_{Z}, R_{\ell} \equiv \Gamma(\mathrm{had}) / \Gamma\left(\ell^{+} \ell^{-}\right), \sigma_{\text {had }} \equiv 12 \pi \Gamma\left(e^{+} e^{-}\right) \Gamma(\mathrm{had}) / M_{Z}^{2} \Gamma_{Z}^{2}, R_{b} \equiv \Gamma(b \bar{b}) / \Gamma(\mathrm{had})$, and $R_{c} \equiv \Gamma(c \bar{c}) / \Gamma$ (had), most of which are weakly correlated experimentally. ( $\Gamma$ (had) is the partial width into hadrons.) The largest correlation coefficient of -0.20 occurs between $R_{b}$ and $R_{c} . R_{\ell}$ is insensitive to $m_{t}$ except for the $Z \rightarrow b \bar{b}$ vertex and final state corrections and the implicit dependence through $\sin ^{2} \theta_{W}$. Thus it is especially useful for constraining $\alpha_{s}$. The width for invisible decays [57], $\Gamma$ (inv) $=\Gamma_{Z}-3 \Gamma\left(\ell^{+} \ell^{-}\right)-\Gamma$ (had $)=500.1 \pm 1.8 \mathrm{MeV}$, can be used to determine the number of neutrino flavors much lighter than $M_{Z} / 2$, $N_{\nu}=\Gamma(\mathrm{inv}) / \Gamma^{\text {theory }}(\nu \bar{\nu})=2.990 \pm 0.011$ for $\left(m_{t}, M_{H}\right)=\left(175 \pm 5 \mathrm{GeV}, M_{Z}\right)$.

There are also measurements of various $Z$ pole asymmetries. These include the polarization or left-right asymmetry

$$
A_{L R} \equiv \frac{\sigma_{L}-\sigma_{R}}{\sigma_{L}+\sigma_{R}},
$$

where $\sigma_{L}\left(\sigma_{R}\right)$ is the cross section for a left- (right)-handed incident electron. $A_{L R}$ has been measured precisely by the SLD collaboration at the SLC [59], and has the advantages of being extremely sensitive to $\sin ^{2} \theta_{W}$ and that systematic uncertainties largely cancel. In addition, the SLD collaboration has extracted the final-state couplings $A_{b}, A_{c}, A_{\tau}$, and $A_{\mu}$ from left-right forward-backward asymmetries [57,60], using

$$
A_{L R}^{F B}(f)=\frac{\sigma_{L F}^{f}-\sigma_{L B}^{f}-\sigma_{R F}^{f}+\sigma_{R B}^{f}}{\sigma_{L F}^{f}+\sigma_{L B}^{f}+\sigma_{R F}^{f}+\sigma_{R B}^{f}}=\frac{3}{4} A_{f},
$$




\section{Electroweak model and constraints on new physics 11}

where, for example, $\sigma_{L F}$ is the cross section for a left-handed incident electron to produce a fermion $f$ traveling in the forward hemisphere. Similarly, $A_{\tau}$ is measured at LEP [57] through the negative total $\tau$ polarization, $\mathcal{P}_{\tau}$, and $A_{e}$ is extracted from the angular distribution of $\mathcal{P}_{\tau}$. An equation such as (10.30) assumes that initial state QED corrections, photon exchange, $\gamma-Z$ interference, the tiny electroweak boxes, and corrections for $\sqrt{s} \neq M_{Z}$ are removed from the data, leaving the pure electroweak asymmetries. This allows the use of effective tree-level expressions,

$$
\begin{gathered}
A_{L R}=A_{e} P_{e}, \\
A_{F B}=\frac{3}{4} A_{f} \frac{A_{e}+P_{e}}{1+P_{e} A_{e}}
\end{gathered}
$$

where

$$
A_{f} \equiv \frac{2 \bar{g}_{V}^{f} \bar{g}_{A}^{f}}{\bar{g}_{V}^{f 2}+\bar{g}_{A}^{f 2}},
$$

and

$$
\begin{aligned}
& \bar{g}_{V}^{f}=\sqrt{\rho_{f}}\left(t_{3 L}^{(f)}-2 q_{f} \kappa_{f} \sin ^{2} \theta_{W}\right), \\
& \bar{g}_{A}^{f}=\sqrt{\rho_{f}} t_{3 L}^{(f)} .
\end{aligned}
$$

$P_{e}$ is the initial $e^{-}$polarization, so that the second equality in Eq. (10.30) is reproduced for $P_{e}=1$, and the $Z$ pole forward-backward asymmetries at LEP $\left(P_{e}=0\right)$ are given by $A_{F B}^{(0, f)}=\frac{3}{4} A_{e} A_{f}$ where $f=e, \mu, \tau, b, c, s$, and $q$, and where $A_{F B}^{(0, q)}$ refers to the hadronic charge asymmetry. The initial state coupling, $A_{e}$, is also determined through the left-right charge asymmetry [61] and in polarized Bhabba scattering [60] at the SLC.

The electroweak-radiative corrections have been absorbed into corrections $\rho_{f}-1$ and $\kappa_{f}-1$, which depend on the fermion $f$ and on the renormalization scheme. In the on-shell scheme, the quadratic $m_{t}$ dependence is given by $\rho_{f} \sim 1+\rho_{t}, \kappa_{f} \sim 1+\rho_{t} / \tan ^{2} \theta_{W}$, while in $\overline{\mathrm{MS}}, \widehat{\rho}_{f} \sim \widehat{\kappa}_{f} \sim 1$, for $f \neq b\left(\widehat{\rho}_{b} \sim 1-\frac{4}{3} \rho_{t}, \widehat{\kappa}_{b} \sim 1+\frac{2}{3} \rho_{t}\right)$. In the $\overline{\mathrm{MS}}$ scheme the normalization is changed according to $G_{F} M_{Z}^{2} / 2 \sqrt{2} \pi \rightarrow \widehat{\alpha} / 4 \widehat{s}_{Z}^{2} \widehat{c}_{Z}^{2}$. (If one continues to normalize amplitudes by $G_{F} M_{Z}^{2} / 2 \sqrt{2} \pi$, as in the 1996 edition of this Review, then $\hat{\rho}_{f}$ contains an additional factor of $\hat{\rho}$.) In practice, additional bosonic and fermionic loops, vertex corrections, leading higher order contributions, etc., must be included. For example, in the $\overline{\mathrm{MS}}$ scheme one has, for $\left(m_{t}, M_{H}\right)=\left(175 \mathrm{GeV}, M_{Z}\right), \widehat{\rho}_{\ell}=0.9978$, $\widehat{\kappa}_{\ell}=1.0013, \widehat{\rho}_{b}=0.9868$ and $\widehat{\kappa}_{b}=1.0067$. It is convenient to define an effective angle $\bar{s}_{f}^{2} \equiv \sin ^{2} \bar{\theta}_{W f} \equiv \widehat{\kappa}_{f} \widehat{s}_{Z}^{2}=\kappa_{f} s_{W}^{2}$, in terms of which $\bar{g}_{V}^{f}$ and $\bar{g}_{A}^{f}$ are given by $\sqrt{\rho_{f}}$ times their tree-level formulae. Because $\bar{g}_{V}^{\ell}$ is very small, not only $A_{L R}^{0}=A_{e}, A_{F B}^{(0, \ell)}$, and $\mathcal{P}_{\tau}$, but also $A_{F B}^{(0, b)}, A_{F B}^{(0, c)}, A_{F B}^{(0, s)}$, and the hadronic asymmetries are mainly sensitive to $\bar{s}_{\ell}^{2}$. One finds that $\widehat{\kappa}_{f}(f \neq b)$ is almost independent of $\left(m_{t}, M_{H}\right)$, so that one can write

$$
\bar{s}_{\ell}^{2} \sim \widehat{s}_{Z}^{2}+0.00029 \text {. }
$$

Thus, the asymmetries determine values of $\bar{s}_{\ell}^{2}$ and $\widehat{s}_{Z}^{2}$ almost independent of $m_{t}$, while the $\kappa$ 's for the other schemes are $m_{t}$ dependent. 